\title{
MBE Growth of CdTe/ZnTe Quantum Dots with Single Mn Ions
}

\author{
K. Gietka, J. Kobak, J.-G. Rousset, E. Janik, T. SŁupiński, P. Kossacki, A. Golnik \\ AND W. PACUSKI \\ Institute of Experimental Physics, Faculty of Physics, University of Warsaw, Hoża 69, 02-681 Warsaw, Poland
}

\begin{abstract}
We report MBE growth and properties of samples with self assembled quantum dots with single manganese ions and low density of quantum dots. Manganese concentration was calibrated using magneto-reflectivity measurements and the giant Zeeman effect in (Cd,Mn)Te and ( $\mathrm{Zn}, \mathrm{Mn}) \mathrm{Te}$ layers. Successful incorporation of $\mathrm{Mn}$ in the $\mathrm{CdTe} / \mathrm{ZnTe}$ quantum dots was confirmed using micro-photoluminescence measurements: single manganese ion in quantum dot manifests in sixfold splitting of exciton emission lines due to $s, p-d$ exchange interaction.
\end{abstract}

PACS: 78.55.Et, 81.07.Ta, 78.67.Hc, 75.50.Pp, 75.75.Cd, 75.30.Hx

\section{Introduction}

The single quantum dots (QDs) containing the single or a few manganese ions [1, 2] are of great interest from point of view of quantum information storage and manipulation [3, 4]. Recently developed technique of the growth of low density self assembled CdTe QDs [5] has led us to grow also such QDs with manganese ions. With ultra low density of QDs finding and isolating dots with single $\mathrm{Mn}$ ion is relatively easy. Moreover, low QDs density opens the possibility of studying not only QDs from low emission energy wing of the QDs spectral distribution, but also other QDs, including typical QDs, with emission energy placed in the middle of such distribution.

\section{Giant Zeeman effect and Mn source calibration}

For the calibration of manganese source we grew series of $(\mathrm{Zn}, \mathrm{Mn}) \mathrm{Te}$ and $(\mathrm{Cd}, \mathrm{Mn}) \mathrm{Te}$ layers with various manganese concentration. Content of manganese was controlled by effusion cell temperature. Samples were grown on GaAs:Si substrates (100) in our new MBE machine (SVT model 35-V-3). They consist of about $100 \mathrm{~nm}$ thick ZnTe buffer layer and $1 \mu \mathrm{m}$ thick CdTe or ZnTe layer doped with manganese. Growth rate was about $600 \mathrm{~nm} / \mathrm{h}$.

In order to examine manganese content we measured polarization resolved reflectivity spectra (Fig. 1a) at low temperatures $(T=1.6 \mathrm{~K})$, and magnetic fields up to $5 \mathrm{~T}$ in the Faraday configuration. All samples exhibit sharp excitonic structures which shifts in the presence of magnetic field due to both the Zeeman and the giant Zeeman effect [6, 7].

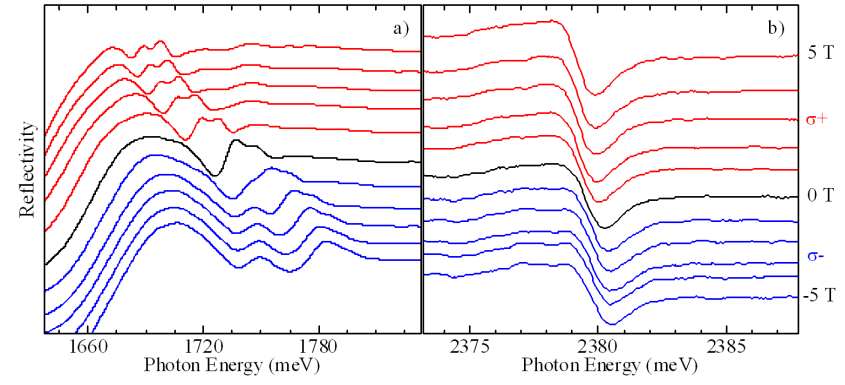

Fig. 1. The giant Zeeman effect: reflectivity spectra of (Cd,Mn) Te (a) and (Zn,Mn)Te (b) measured in Faraday configuration, at magnetic fields up to $5 \mathrm{~T}$.

The giant Zeeman effect is proportional to manganese concentration thus we were able to determine manganese concentration. For (Cd,Mn)Te sample we simply fitted modified Brillouin function [6] to experimental data with a manganese concentration as a fitting parameter. We obtained the concentration $x_{\mathrm{Mn}}=8 \%$ (Fig. 2) for the giant Zeeman splitting of about $100 \mathrm{meV}$, which is two orders of magnitude larger than normal Zeeman effect, so we neglected the former one in this analysis. Similar procedure was used for the analysis of the (Zn,Mn)Te with concentration $x_{\mathrm{Mn}}=6 \%$ and $x_{\mathrm{Mn}}=1 \%$ (not shown). The exchange parameters were taken from Ref. [7].

For very diluted (Zn,Mn)Te sample (Fig. 1b) manganese concentration was obtained by comparing (Zn,Mn)Te exciton splitting to the result obtained for reference ZnTe layer (Fig. 3).

Blue curve in Fig. 3a represents $a B^{2}+b B+c$ fit where $a, b$, and $c$ denote diamagnetic shift, Zeeman shift and zero field energy, respectively $\left(a=0.0157 \mathrm{meV} / \mathrm{T}^{2}\right.$, $b=0.0581 \mathrm{meV} / \mathrm{T}, c=2379.8 \mathrm{meV})$. Parabola fit- 


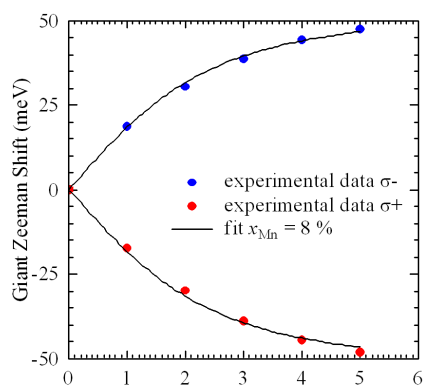

Fig. 2. Giant Zeeman shift versus magnetic field for $\mathrm{Cd}_{1-x} \mathrm{Mn}_{x}$ Te. Points represent experiment related to spectra shown in Fig. 1a. Line represents modified Brillouin fit with parameter $x=8 \%$.
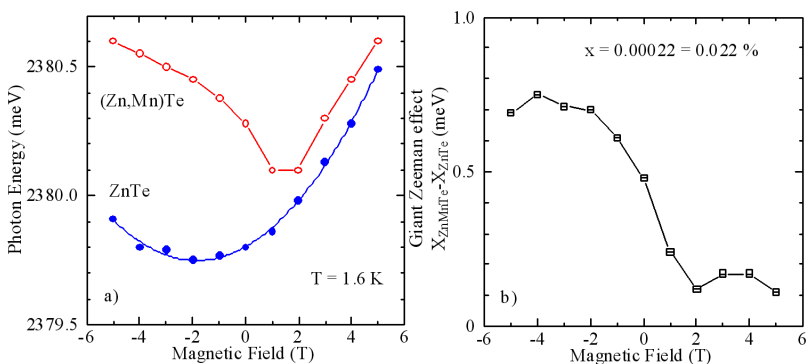

Fig. 3. Position of excitons versus magnetic field. (a) Reference ZnTe sample - blue (bottom) curve and (Zn,Mn)Te sample - red (topmost). (b) The difference between position of excitons in $(\mathrm{Zn}, \mathrm{Mn}) \mathrm{Te}$ and $\mathrm{ZnTe}$, which is interpreted as the giant Zeeman effect in $(\mathrm{Zn}, \mathrm{Mn}) \mathrm{Te}$.

ted to $\mathrm{ZnTe}$ data was subtracted from $(\mathrm{Zn}, \mathrm{Mn}) \mathrm{Te}$ data. The result is shown in Fig. 3b. Such a procedure revealed us pure manganese influence and therefore enabled to determine very low concentration of manganese $(x=0.022 \pm 0.005 \%)$. The source calibration for the lowest Mn concentration was used for further growth of magnetic quantum dots.

\section{QDs growth}

We have made two quantum dots samples, which were grown using a method of amorphous tellurium [8] on GaAs:Si substrate (100). Samples differ in concentration of manganese. In order to build in the manganese in quantum dot structure [2], during the deposition of CdTe we opened the manganese effusion cell for three seconds. Temperature of manganese source was $590^{\circ} \mathrm{C}$ and $620^{\circ} \mathrm{C}$ for consecutive samples (temperature measured with thermocouple). Mn flux used for sample grown with Mn source at $590{ }^{\circ} \mathrm{C}$ corresponds to $x_{\mathrm{Mn}}=0.0022 \%$ [in $(\mathrm{Zn}, \mathrm{Mn}) \mathrm{Te}$ layer].

\section{Microphotoluminescence}

The optical properties of quantum dots were studied using microphotoluminescence at $1.6 \mathrm{~K}$, excited by blue laser diode (403 nm). All samples exhibit sharp photoluminescence lines characteristic for quantum dots (Fig. 4). We observed that quantum dots density in samples with manganese is one order of magnitude lower than in the reference sample grown in the same conditions before, without Mn deposition. The decrease of the QDs density was described by Kuroda et al. [9] and was attributed to the reduced mobility of CdTe on ZnTe in the presence of Mn ions. For the first sample with Mn quantum dots density is $c a .50$ dots per $\mu \mathrm{m}^{2}$ and for the second sample with Mn quantum dots density is $c a .30$ dots per $\mu \mathrm{m}^{2}$ (one or two orders of magnitude lower density than e.g. in various samples presented in Ref. [10]). With such low density of quantum dots it is relatively easy to find them with single manganese ion.

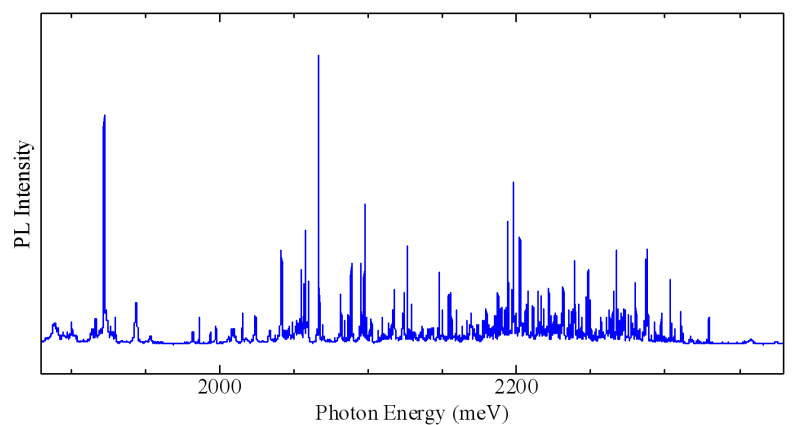

Fig. 4. Microphotoluminescence spectra of $\mathrm{CdTe} / \mathrm{ZnTe}$ QDs ensemble. Some of QDs contain $\mathrm{Mn}$ ions. Sample with lower concentration of Mn.
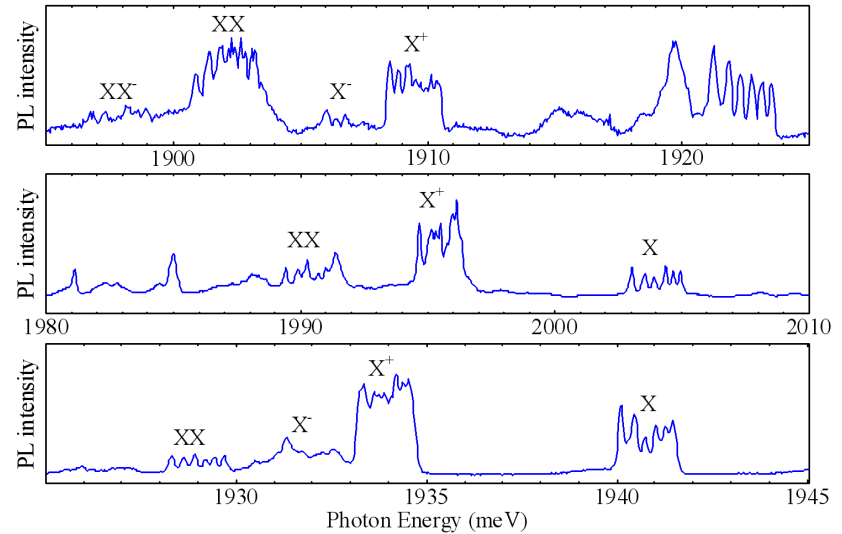

Fig. 5. Quantum dots emission lines split due to the exchange interaction with single $\mathrm{Mn}$ ion. There are lines related to excitons $(\mathrm{X})$, trions $\left(\mathrm{X}^{+}\right.$and $\left.\mathrm{X}^{-}\right)$, biexcitons $(\mathrm{XX})$, and charged biexcitons $\left(\mathrm{XX}^{-}\right)$.

As seen in Fig. 4 in the low energy region we observe broader lines than in high energy region. We interpret this effect as a result of the presence of a few manganese ions in large dots [2]. Due to quantum confinement large dots have lower energy than small dots. Manganese ions are more likely to arrange in dots with more space within. 
We identified also quantum dot emission lines split into 6 (Fig. 5) due to exchange interaction with the single $\mathrm{Mn}$ ion [1]. We estimated that for the sample with lower content of manganese the highest probability of finding a dot with single manganese ion is in the energy range from $1900 \mathrm{meV}$ up to $2000 \mathrm{meV}$.

\section{Conclusions}

Giant Zeeman effect was used in order to determine very low $M n$ concentration (down to $x=0.022 \%$ ) and to calibrate Mn molecular flux useful for growth of QDs with single Mn. We obtained simple growth scheme of quantum dots with single magnetic ions. Resulting QDs have low density which makes finding of QDs with single magnetic ion relatively easy.

\section{Acknowledgments}

We wish to acknowledge helpful discussions with Piotr Wojnar. This work was supported by Polish public funds in years 2011-2017 (NCN projects DEC-2011/ 01/B/ST3/02406 and DEC-2011/02/A/ST3/00131 and NCBiR project LIDER/30/13/L-2/10/NCBiR/ 2011). Project was carried out with the use of CePT, CeZaMat and NLTK infrastructures financed by the European Union - the European Regional Development Fund within the Operational Programme "Innovative economy" for 2007-2013.

\section{References}

[1] L. Besombes, Y. Léger, L. Maingault, D. Ferrand, H. Mariette, J. Cibert, Phys. Rev. Lett. 93, 207403 (2004).

[2] P. Wojnar, J. Suffczyński, K. Kowalik, A. Golnik, G. Karczewski, J. Kossut, Phys. Rev. B 75, 155301 (2007).

[3] C. Le Gall, L. Besombes, H. Boukari, R. Kolodka, J. Cibert, H. Mariette, Phys. Rev. Lett. 102, 127402 (2009).

[4] M. Goryca, T. Kazimierczuk, M. Nawrocki, A. Golnik, J.A. Gaj, P. Kossacki, P. Wojnar, G. Karczewski, Phys. Rev. Lett. 103, 087401 (2009).

[5] J. Kobak, J.-G. Rousset, R. Rudniewski, E. Janik, Słupiński, P. Kossacki, A. Golnik, W. Pacuski, eprint arXiv:1210.2946.

[6] J.A. Gaj, W. Grieshaber, C. Bodin-Deshayes, J. Cibert, G. Feuillet, Y. Merle d'Aubigné, A. Wasiela, Phys. Rev. B 50, 5512 (1994).

[7] A. Twardowski, P. Swiderski, M. von Ortenberg, R. Pauthenet, Solid State Commun. 50, 509 (1984).

[8] F. Tinjod, S. Moehl, K. Kheng, B. Gilles, H. Mariette, Appl. Phys. Lett. 82, 4340 (2003).

[9] S. Kuroda, Y. Terai, K. Takita, T. Takamasu, G. Kido, N. Hasegawa, T. Kuroda, F. Minami, J. Crystal Growth 214\&215, 140 (2000).

[10] J. Kobak, W. Pacuski, T. Jakubczyk, T. Kazimierczuk, A. Golnik, K. Frank, A. Rosenauer, C. Kruse, D. Hommel, J.A. Gaj, Acta Phys. Pol. A 119, 627 (2011). 